\title{
Approach to contraception in the primary health care: barrier, behavioral and pharmaceutical methods
}

\author{
Shada Omar Baoum ${ }^{1 *}$, Zainab Abduljabbar Almomen², Fatmah Abdullah Alotaibi, \\ Rawan Ayedh Almohaiya ${ }^{4}$, Amani Abdullah Alharbi ${ }^{5}$, Hamed Arif Hejazi ${ }^{6}$, \\ Hibah Abdullah Alsabei ${ }^{7}$, Norah Salem Algethami ${ }^{8}$, Nareman Sulaiman Shamlan', \\ Norah Dakhilallah Aljohani ${ }^{10}$, Nura Ayidh Alqahtani ${ }^{3}$
}

\author{
${ }^{1}$ Primary Healthcare, King Fahad General Hospital, Jeddah, Saudi Arabia \\ ${ }^{2}$ College of Medicine, Imam Abdulrahman Bin Faisal University, Dammam, Saudi Arabia \\ ${ }^{3}$ College of Medicine, King Saud Bin Abdulaziz University for Health Sciences, Riyadh, Saudi Arabia \\ ${ }^{4}$ Department of Obstetrics and Gynecology, King Saud Medical City, Riyadh, Saudi Arabia \\ ${ }^{5}$ College of Medicine, Almaarefa Colleges, Riyadh, Saudi Arabia \\ ${ }^{6}$ Department of Orthopaedics, Al Noor Specialist Hospital, Mecca, Saudi Arabia \\ ${ }^{7}$ Primary Healthcare Center, Ministry of Health, Dammam, Saudi Arabia \\ ${ }^{8}$ College of Medicine, Umm Al-Qura University, Mecca, Saudi Arabia \\ ${ }^{9}$ Department of Obstetrics and Gynecology, King Abdullah Hospital, Jeddah, Saudi Arabia \\ ${ }^{10}$ College of Medicine, Taibah University, Medina, Saudi Arabia
}

Received: 25 October 2021

Accepted: 11 November 2021

*Correspondence:

Dr. Shada Omar Baoum,

E-mail: baoum.shadaa@gmail.com

Copyright: (c) the author(s), publisher and licensee Medip Academy. This is an open-access article distributed under the terms of the Creative Commons Attribution Non-Commercial License, which permits unrestricted non-commercial use, distribution, and reproduction in any medium, provided the original work is properly cited.

\begin{abstract}
Contraception is a critical tool in achieving favorable family planning, which has been reported with remarkable outcomes on the individual and country-based levels. Using contraceptive approaches has been reported to be cost efficacious with minimal side effects. It gives couples more freedom, privacy and control over their lives, which might also enhance the quality of life of these individuals. In the present study, we have reviewed evidence from current studies in the literature about the different approaches to contraception in primary care. We have discussed the different barrier and non-barrier approaches that have been validated in the literature as efficacious approaches that can achieve contraception. We also reviewed the safety profiles for the different modalities and whether they can be used or not. Overall, couples should consult with the primary care physician before approaching any modality to give them a better judgment about the potential benefits and risks of each suitable contraception tool. We suggest that educational campaigns should also be conducted to increase awareness and attitude about family planning and using contraceptive modalities to expand the application and favorable outcomes of using these tools.
\end{abstract}

Keywords: Contraception, Barrier, Non-barrier, Pharmaceutical, Oral contraceptive pills

\section{INTRODUCTION}

Contraception is known as performing intentional approaches to prevent conception by using different modalities including barrier, behavioral and pharmaceutical tools, in addition to some surgical procedures. ${ }^{1}$ Contraception is a critical tool in achieving favorable family planning, which has been reported with remarkable outcomes on the individual and country-based levels. $^{2}$ This can allow couples to enjoy their physical lives with limited fear of having undesired children and giving them more freedom to decide when to get pregnant and have children. 
Using contraceptive approaches has been reported to be cost efficacious with minimal side effects. Besides, it gives couples more freedom, privacy and control over their lives, which might also enhance the quality of life of these individuals. Furthermore, some tools can effectively intervene against catching certain infections (as sexually transmitted diseases), which adds to its advantageous list. ${ }^{3} \mathrm{We}$ aimed to conduct a literature review to discuss the different approaches to contraception in primary care settings including barrier, pharmaceutical and behavioral approaches.

\section{Methods}

To retrive relevant studies, we conducted an extensive literature search of the Medline, Cochrane and EMBASE databases which was performed on 27 October 2021 using the medical subject headings (MeSH) or a combination of all possible related terms, according to the database. To avoid missing potential studies, a further manual search for papers was done through Google Scholar, while the reference lists of the initially included papers. Studies discussing approach to contraception in the primary health care were screened for useful information, with no limitations posed on date, language, age of participants or publication type. Only Saudi-based studies were included.

\section{DISCUSSION}

\section{Pharmaceutical methods}

Different modalities have been reported in the literature as effective approaches to achieve contraception. In the present section, we will shed more light on the different barrier, behavioral and pharmaceutical methods to achieve contraception in primary care settings and the different barriers to adequately achieve these approaches and obtain favorable outcomes.

\section{Oral and injectable contraceptives}

Oral contraceptive pills have been used with great outcomes, according to the different studies. It has been demonstrated that the estimated efficacy of these modalities might be up to $99 \%$ when used properly. ${ }^{4}$ The amount of estrogen within these modalities usually ranges between 20 and $40 \mu \mathrm{g}$. On the other hand, based on the potency of these modalities, it has been demonstrated that the amount of progestin can significantly differ. Minipill or progestin-low dose products were also special forms of oral contraceptive pills that were also reported in the literature and validated for use in these settings. Many benefits were observed among women receiving hormonal combinations of contraceptive pills. However, whatever these benefits were, the administration of contraceptive pills should only be approached after adequate counseling with the healthcare professional to determine the potential risks and benefits from receiving these modalities and whether they can be helpful to these women or not. Among the reported benefits, previous reports also indicated that the risk of developing ovarian cancer was significantly reduced by $40 \%$ in women that received these modalities at any stage of their menstrual life. After 10 years of using these modalities, further evidence showed that the risk was even shortened up to $80 \%$. Favorable protection efficacies were also reported for endometrial cancer. ${ }^{5}$ Reports showed that the use of these modalities for up to 15 years can significantly reduce the risk of developing these cancers up to a great extent. The protective effects were also reported for breast cancer and the impact was reported to last for one year after discontinuing the administration of oral contraceptive pills. ${ }^{6}$ Although many favorable events were reported for these modalities, evidence showed that there were some side effects for using them. Nonetheless, it should be noted that the incidence of these events had been greatly reduced as a result of the great advances in the field and the use of low dose formulations. For instance, many cardiovascular events were reported for women receiving oral contraceptive pills. ${ }^{7}$ However, it should be noted that these modalities were significantly based on the presence of risk factors of these diseases and how frequent patients were exposed to them. Previous reports showed that using low dose estrogen was significantly associated with a reduced risk of developing ischemic stroke in patients with hypertension and smokers. ${ }^{7,8}$ Migraine and thromboembolism were also reported to increase following the administration of these modalities. Nevertheless, it had been reported that the incidence significantly decreased when using low doses. Some investigations also reported remarkable concerns about the incidence of breast cancer as estimated show that the incidence can increase within 10 years after drug discontinuation. However, it had been furtherly reported that this risk did not further increase following these 10 years. ${ }^{9-11}$ Self-examination by the women at high risk was then mandatory for achieving favorable outcomes and enhancing the prognosis.

Medroxyprogesterone acetate had been validated as an efficacious injectable formulation that was derived from progesterone to prevent pregnancy. Nonetheless, it should be noted that 2 main side events were reported for this modality including a delayed return to normal fertility after discontinuation of administration and remarkable menstrual changes. ${ }^{12,13}$ It had been demonstrated that the reported delay might last for up to 24 months. Nevertheless, reports also indicated that most women were usually pregnant by the end of one year. Weight gain was also reported as a potential side effect after using these modalities. Therefore, consultation with the primary healthcare physician about the potential development of these adverse events was essential before starting administration. ${ }^{14,15}$

\section{Subdermal implants and transdermal patches}

Long term contraception was also reported to be significantly achieved by using subdermal implant rods 
that can remarkably release sufficient amounts of etonogestrel that can reduce contraception. Estimates showed that this approach was probably the most efficacious modality and studies showed that the potential failure rate of contraception had been reported to be $0.05 \%$ only. ${ }^{16,17}$ The main advantage of using these modalities and the reported high efficacy was the easy installment within the patients' bodies. It can be installed using only local anesthesia in a minimally invasive procedure within an estimated duration of 5 minutes by the attending physician, unlike the insertion of a device that needed surgical interference. It had been furtherly reported that it can be easily removed at any time based on the patient's needs. However, some reports suggested that it should be continued for three years and after which ovulation can normally continue within 3-4 weeks following discontinuation. Besides, the incidence of complications has been reported to be very low regarding the insertion and removal process. ${ }^{18,19}$ These complications were not serious and included hematoma formation, local infections, allergic reactions and irritation. Furthermore, the modality was generally recommended for patients with an increased risk of developing hypertension, thromboembolism, obesity and other patients who were $>35$ years old and smokers, that were not encouraged to receive contraceptives that contained estrogen as previously discussed. Pregnancy, hypersensitivity to any components of the modality and having breast cancers were all absolute contraindications of using the subdermal implant. On the other hand, evidence indicated that the administration of these modalities was safe for lactating women and can be administered within the postpartum period. The main side effect that was usually encountered following the use of subdermal implants was irregular bleeding. Weight gain, infections, pain at the site of insertion, local scarring, discoloration of the skin, skin irritation and potential changes in sex drive were also other adverse events reported for these modalities. ${ }^{14,20,21}$

Synthetic ethinyl estradiol and norelgestromin can be delivered in a transdermal patch and have been validated as efficacious modalities that can prevent contraception. These patches were recommended to be applied over the upper outer arm, buttocks, back or abdomen in areas that were clean, dry, non-irritated and intact. ${ }^{22}$ Not using oils, powders, lotions and creams at the site of administration was also recommended because this might influence the patch to fall. However, it should be noted that proper application of the modality allowed patients to presume their daily routine activities including showering and swimming, with no interruption of the mechanism of action of the patch. ${ }^{23}$ It should be recommended that patients should check the position of the patch daily to maintain it was in its right position to maintain good outcomes. Breast discomfort, dysmenorrhea and headache were the main side effects reported for this modality and the potential to develop the aforementioned adverse events that were reported with oral and injectable contraceptive combinations..$^{23,24}$

\section{Intravaginal rings and intrauterine devices}

Using intravaginal rings was also validated by previous research. These modalities were usually used to deliver etonogestrel/ethinyl estradiol in a safe practice. Headache had been reported as the most frequent adverse event, with an estimated rate of $7 \%$. Nevertheless, some reports showed that device expulsion, coital conditions, weight gain, nausea and leucorrhea were other adverse events that can be reported by using women. ${ }^{25-27}$ In a similar context, using intrauterine devices was also reported to be efficacious in these settings and evidence showed that they can work with and without hormones. It had also been reported that the modality was not contraindicated during lactation and can be administered during breastfeeding within the postpartum period. ${ }^{28}$ Headache, ovarian cysts, acne, pelviabdominal pain, vulvovaginitis and alternations in the bleeding patterns were the commonest adverse events that were reported for these modalities. ${ }^{29-32}$ Perforation of the cervix or ureteric wall, pelvic infection, sepsis, intrauterine pregnancy and ectopic pregnancy were also other adverse events that can potentially develop following the installation of this modality. ${ }^{33}$ These adverse events can be significantly reduced in non-hormonal dependant devices. However, it should be noted that some other adverse events were also reported for these modalities including heavier periods, increased incidence of pelvic inflammatory diseases, perforation of the cervix or uterine wall and device embedment. ${ }^{34}$ It should be noted that increased cardiovascular risk was another disadvantage of these modalities due to the effect of hormonal combinations. Placement-related complications, infections and sensitivities can also be frequently encountered in the non-hormonal dependant modalities..$^{9-11}$

\section{Barrier and behavioral methods}

Other methods of contraception included non-barrier and behavioral approaches. For instance, using male condoms had been reported to be frequent across the different settings of contraception. These modalities can also provide acceptable protection against different sexually transmitted diseases and being easy to use. ${ }^{35}$ Different forms of condoms were reported in the literature and they usually vary based on the structure as wanted function as required by the patient. ${ }^{36,37}$ Vaginal condoms were also other barrier modalities that can be used to effectively reduce the incidence of contraception. ${ }^{38}$ Their structure differed from that of men and was vaginally inserted. It can also be used as a spermicide modality and therefore, it had been reported as an efficacious chemical and physical contraceptive barrier. Using male condoms can also be simultaneous with the use of these modalities. However, it should be noted that these approaches should not be used for more than 6 hours. Besides, some complications might occur including incompatible bladder emptying and urinary tract infections. The risk of toxic shock syndrome has been reported to be associated with cases when the diaphragm has been left within the 
vagina for more than 24 hours. ${ }^{39}$ Using a cervical cap has also been validated in the different studies in the literature. These modalities can be inserted for long periods (at least for 6 hours after intercourse and 42 hours before it), with a more reduced risk of developing toxic shock syndrome than the reported risk with the diaphragm. ${ }^{40-42}$ Having cervical cancer and abnormally shaped cervix were contraindications for using this modality. Studies also validated the use of spermicidal formulas. Nevertheless, evidence indicated that they have low efficacy and can cause irritation to the penis and vagina. ${ }^{43}$ Family planning and counseling were also reported as favorable approaches that can enhance the outcomes and achieve contraception according to many previous investigations. ${ }^{44}$

\section{CONCLUSION}

Overall, couples should consult with the primary care physician before approaching any modality to give them a better judgment about the potential benefits and risks of each suitable contraception tool. We suggest that educational campaigns should also be conducted to increase awareness and attitude about family planning and using contraceptive modalities to expand the application and favorable outcomes of using these tools.

Funding: No funding sources

Conflict of interest: None declared

Ethical approval: Not required

\section{REFERENCES}

1. Jain R, Muralidhar S. Contraceptive methods: needs, options and utilization. J Obstetr Gynaecol India. 2011;61(6):626-34.

2. Colquitt CW, Martin TS. Contraceptive methods. J Pharma Pract. 2017;30(1):130-5.

3. Bromham DR. STD prevention and unplanned pregnancies in Great Britain. Contracepti Fertilit Sexualit. 1995;23(4):255-7.

4. Stricker R, Eberhart R, Chevailler MC, Quinn FA, Bischof P, Stricker R. Establishment of detailed reference values for luteinizing hormone, follicle stimulating hormone, estradiol, and progesterone during different phases of the menstrual cycle on the Abbott ARCHITECT analyzer. Clinic Chem Lab Med. 2006;44(7):883-7.

5. Breast cancer and hormonal contraceptives: further results. Collaborative Group on Hormonal Factors in Breast Cancer. Contraception. 1996;54(3):1-106.

6. Grabrick DM, Hartmann LC, Cerhan JR, Vierkant RA, Therneau TM, Vachon CM, et al. Risk of breast cancer with oral contraceptive use in women with a family history of breast cancer. JAMA. 2000;284(14):1791-8.

7. Ness RB, Grisso JA, Klapper J, Schlesselman JJ, Silberzweig S, Vergona R, et al. Risk of ovarian cancer in relation to estrogen and progestin dose and use characteristics of oral contraceptives. SHARE study group. Steroid Hormones and reproductions. Am J Epidemiol. 2000;152(3):233-41.

8. Sulak PJ. Oral contraceptives: therapeutic uses and quality-of-life benefits-case presentations. Contraception. 1999;59(1):35-8.

9. Rosenberg L, Palmer JR, Sands MI, Grimes D, Bergman U, Daling J, et al. Modern oral contraceptives and cardiovascular disease. Am J Obstetr Gynecol. 1997;177(3):707-15.

10. Consensus conference on combination oral contraceptives and cardiovascular disease. Fertil Steril. 1999;71:1-6.

11. Chen FC, vonDehn D, Büscher U, Dudenhausen JW, Niggemann B. Atopy, the use of condoms, and a history of cesarean delivery: potential predisposing factors for latex sensitization in pregnant women. Am J Obstetr Gynecol. 1999;181(6):1461-4.

12. Schivone G, Dorflinger L, Halpern V. Injectable contraception: updates and innovation. Curr Opin Obstetr Gynecol. 2016;28(6):504-9.

13. Upadhyay UD, Zlidar VM, Foster DG. Interest in self-administration of subcutaneous depot medroxyprogesterone acetate in the United States. Contraception. 2016;94(4):303-13.

14. Trussell J, Guthrie K. Choosing a contraceptive: Efficacy, safety, and personal considerations. Contracept Technol. 2007:45-74.

15. Trussell J. Contraceptive failure in the United States. Contraception. 2011;83(5):397-404.

16. Hohmann H, Creinin MD. The contraceptive implant. Clinic Obstetr Gynecol. 2007;50(4):907-17.

17. Etonogestrel implant (Implanon) for contraception. Drug Therapeut Bull. 2001;39(8):57-9.

18. Darney PD. Implantable contraception. Eur J Contracept Reproduct Health Care. 2000;5(2):2-11.

19. Funk S, Miller MM, Mishell DR, Archer DF, Poindexter A, Schmidt J, et al. Safety and efficacy of Implanon, a single-rod implantable contraceptive containing etonogestrel. Contraception. 2005;71(5):319-26.

20. Ali M, Bahamondes L, Landoulsi S. Extended effectiveness of the etonogestrel-releasing contraceptive implant and the $20 \mu \mathrm{g}$ levonorgestrelreleasing intrauterine system for 2 years beyond U.S. food and drug administration product labeling. Glob Health Sci Pract. 2017;5(4):534-9.

21. McNicholas C, Swor E, Wan L, Peipert JF. Prolonged use of the etonogestrel implant and levonorgestrel intrauterine device: 2 years beyond food and drug administration-approved duration. Am J Obstetr Gynecol. 2017;216(6):586.

22. Abrams LS, Skee DM, Natarajan J, Wong FA, Anderson GD. Pharmacokinetics of a contraceptive patch (Evra/Ortho Evra) containing norelgestromin and ethinyloestradiol at four application sites. $\mathrm{Br} \mathrm{J}$ Clin Pharmacol. 2002;53(2):141-6.

23. Parasrampuria DA, Vaughan S, Ariyawansa J, Swinnen A, Natarajan J, Rasschaert F, et al. Comparison of a transdermal contraceptive patch with a newly sourced adhesive component versus 
EVRA patch: a double-blind, randomized, bioequivalence and adhesion study in healthy women. Contraception. 2020;101(4):276-82.

24. Gruber D, Skřivánek A, Serrani M, Lanius V, Merz M. A comparison of bleeding patterns and cycle control using two transdermal contraceptive systems: a multicenter, open-label, randomized study. Contraception. 2015;91(2):105-12.

25. Roumen F. Contraceptive efficacy and tolerability with a novel combined contraceptive vaginal ring, NuvaRing. Eur J Contracept Reproduct Health Care. 2002;7(2):19-24.

26. Ethinylestradiol+etonogestrel contraceptive vaginal ring: new drug. Possibly useful in some situations. Prescrire Int. 2006;15(82):50-3.

27. Bruni V, Pontello V, Luisi S, Petraglia F. An openlabel, multicentre trial to evaluate the vaginal bleeding pattern of the combined contraceptive vaginal ring NuvaRing. Eur J Obstetr Gynecol Reproduct Biol. 2008;139(1):65-71.

28. Kerns J, Darney P. Vaginal ring contraception. Contraception. 2011;83(2):107-15.

29. Odlind V. Modern intra-uterine devices. Bailliere Clinic Obstetr Gynaecol. 1996;10(1):55-67.

30. Monaco J, Zolotor A. Optimal use of IUDs: why aren't we there yet? Am Fam Physician. 2014;89(6):434.

31. Intrauterine devices: an effective alternative to oral hormonal contraception. Prescrire Int. 2009;18(101):125-30.

32. Fortney JA, Feldblum PJ, Raymond EG. Intrauterine devices. The optimal long-term contraceptive method? J Reproduct Med. 1999;44(3):269-74.

33. Hardeman J, Weiss BD. Intrauterine devices: an update. Am Fam Physician. 2014;89(6):445-50.

34. Edwardson J, Jamshidi R. The contraceptive vaginal ring. Semin Reproduct Med. 2010;28(2):133-9.

35. Walsh TL, Frezieres RG, Peacock K, Nelson AL, Clark VA, Bernstein L. Evaluation of the efficacy of a nonlatex condom: results from a randomized, controlled clinical trial. Perspect Sex Reproduct Health. 2003;35(2):79-86.
36. Gallo MF, Grimes DA, Schulz KF. Nonlatex vs. latex male condoms for contraception: a systematic review of randomized controlled trials. Contraception. 2003;68(5):319-26.

37. Steiner MJ, Dominik R, Rountree RW, Nanda K, Dorflinger LJ. Contraceptive effectiveness of a polyurethane condom and a latex condom: a randomized controlled trial. Obstetr Gynecol. 2003;101(3):539-47.

38. McNamee K. The female condom. Austral Fam Physician. 2000;29(6):555-7.

39. Trussell J, Strickler J, Vaughan B. Contraceptive efficacy of the diaphragm, the sponge and the cervical cap. Fam Plan Perspect. 1993;25(3):100-5.

40. Richardson BA. Nonoxynol-9 as a vaginal microbicide for prevention of sexually transmitted infections: it's time to move on. JAMA. 2002;287(9):1171-2.

41. Secor RM. The cervical cap. NAACOG Clinic Issue Perinat Women Health Nurs. 1992;3(2):236-45.

42. Smith M, Barwin BN. Vaginal mechanical contraceptive devices. Canad Med Assoc J. 1983;129(7):699-701.

43. Wilcox AJ, Dunson DB, Weinberg CR, Trussell J, Baird DD. Likelihood of conception with a single act of intercourse: providing benchmark rates for assessment of post-coital contraceptives. Contraception. 2001;63(4):211-5.

44. Lopez LM, Grey TW, Chen M, Denison J, Stuart G. Behavioral interventions for improving contraceptive use among women living with HIV. Coch Database Systemat Rev. 2016;2016(8):010243.

Cite this article as: Baoum $\mathrm{SO}$, Almomen $\mathrm{ZA}$, Alotaibi FA, Almohaiya RA, Alharbi AA, Hejazi HA, et al. Approach to contraception in the primary health care: barrier, behavioral and pharmaceutical methods. Int J Community Med Public Health 2021;8:6122-6. 